Gynäkologische Endokrinologie 2014 · 12:51-54

DOI 10.1007/s10304-013-0612-0

Online publiziert: 4. Januar 2014

c) Springer-Verlag Berlin Heidelberg 2014

\section{M. von Wolff $\cdot$ P. Stute}

Abteilung für Gynäkologische Endokrinologie und Reproduktionsmedizin,

Universitäts-Frauenklinik, Inselspital Bern, Bern

\title{
Neues und praktisch Relevantes aus der gynäkologischen Endokrinologie
}

\section{Weltkongresse 2013}

Für Sie besucht, kondensiert und präsentiert

Am 5. September 2013 fand zum zweiten Mal in der Schweiz eine Fortbildung zum Themenbereich der gynäkologischen Endokrinologie und Reproduktionsmedizin statt, bei der die Kongress-Highlights des letzten Jahres zusammengefasst und Neues sowie Praxisrelevantes von M. von Wolff und P. Stute vorgestellt wurde. Wie schon im ersten Jahr lag der Schwerpunkt auf Themen der gynäkologischen Endokrinologie. Alle Beiträge können inzwischen auf der Website (http://www.weltkongresse.de, http:// www.weltkongresse.ch und http://www. weltkongresse.at) abgerufen werden. Im Folgenden werden neun der referierten Kongress-Highlights zum Themenbereich der gynäkologischen Endokrinologie kurz dargestellt.

\section{Deutsche Gesellschaft für Endokrinologie (DGE)}

\section{Chronopharmakologie und -biologie - bisher wenig beachtet, aber klinisch relevant}

Der Stoffwechsel der Organe muss sich an ständig wechselnde Umwelteinflüsse anpassen. Viele Organe sind tagsüber aktiver, andere nachts. Dies wird ermöglicht durch zeitliche Taktgeber, die in fast allen Organen zu finden sind. Die Synchronisation mit den Umwelteinflüssen erfolgt durch molekulare Uhrwerke, die aus Proteinen bestehen, die in regelmäßigen Abständen vermehrt und vermindert synthetisiert werden. Das gesamte System funktioniert wahrscheinlich wie ein Orchester. Die Organe (Musiker) haben einen eigenen Rhythmus, den der vom Licht beeinflusste hypothalamische $\mathrm{Nu}$ cleus suprachiasmaticus (Dirigent) über das vegetative Nervensystem und endokrin, z. B. über die Kortisolproduktion der Nebenniere, harmonisiert.

> Die klinische Relevanz der Chronobiologie zeigt sich in dem jungen Forschungszweig der Chronopharmakologie.

Medikamente wie Schlafmittel können chronobiologische Prozesse stören, andere, z. B. Chronobiotika wie Melatonin, synchronisieren sie. Da Medikamente je nach Aktivität eines Organs unterschiedlich aufgenommen und metabolisiert werden und somit ihre Wirkung chronobiologisch beeinflusst wird, sind Kenntnisse im Bereich der Chronobiologie und -pharmakologie wichtig, aber erst in Ansätzen vorhanden.

Ein klinisch-praktisches Beispiel ist die Einnahme von Schilddrüsenhormonen, die üblicherweise morgens und mindestens 30 min vor dem Frühstück erfolgen soll, um die Aufnahme des Medikaments zu maximieren. Chronopharmakologisch wäre aber wahrscheinlich eine abendliche Gabe sinnvoller, da nachts u. a. die Darmmotilität reduziert und somit die Aufnahme verbessert ist [5].
Fazit

Kenntnisse über die Chronobiologie und Chronopharmakologie sind klinisch relevant, aber bislang nur begrenzt vorhanden. Eine Veränderung des Tag-NachtRhythmus kann zu Veränderungen in der Wirksamkeit von Medikamenten führen.

Initiierung der Ovulation und zirkadiane Ausschüttung von Kortisol

Bislang ist unklar, wann im Laufe des Tages die Ovulation geschieht. Da der Geschlechtsverkehr nicht erst nach der Ovulation erfolgen sollte, sind Fragen von $\mathrm{Pa}$ tientinnen zu dieser Thematik nicht selten. Erwiesen ist, dass die Ovulation durch die Zunahme der Konzentration des luteinisierenden Hormons (LH) initiiert wird. Der LH-Anstieg scheint an den Anstieg der Kortisolkonzentration gekoppelt zu sein. Bei Frauen mit einem Kortisol-Peak in den frühen Morgenstunden steigt LH meist früher an als bei einem Maximum in den späteren Morgenstunden [6]. Grundsätzlich bedeutet dies, dass die LH-Zunahme meist in den frühen Morgenstunden beginnt. Der zeitliche Abstand zwischen dem Einsetzen des LH-Anstiegs und der Ovulation ist nicht sicher bekannt. Gemäß einer Studie der Weltgesundheitsorganisation (WHO) aus den 1980er-Jahren [16] ereignet sich die Ovulation in 90\% der Fälle 23,6-38,2 h (Median: 32,0 h) nach dem Beginn des LH-Anstiegs. Allerdings sind diese Daten aufgrund des Studiendesigns nicht sehr genau. Legt man diese Daten zugrunde, 
erfolgt die Ovulation natürlicherweise meist im Laufe des Tages und mit der höchsten Wahrscheinlichkeit im Laufe des Vormittags, nicht aber in der Nacht.

\section{Fazit}

Die Initiierung der Ovulation ist vermutlich an die zirkadiane Rhythmik der Kortisolsekretion gekoppelt. Die Ovulation erfolgt wahrscheinlich am häufigsten tagsüber. Eine klinische Konsequenz hinsichtlich des besten Zeitpunkts für den Geschlechtsverkehr kann jedoch nicht abgeleitet werden.

\section{Schilddrüsenhormonsubstitution bei Kinderwunschpatientinnen}

Bei einem Kinderwunsch wird inzwischen häufig eine Schilddrüsenhormonsubstitution durchgeführt. Der Grund hierfür sind Berichte, dass der obere Grenzwert des thyroideastimulierenden Hormons (TSH), der (laborabhängig) bei etwa 4,0 mU/l liegt, bei einem Kinderwunsch und insbesondere bei einer Sterilität gesenkt werden sollte. Als Zielgröße werden $2,5 \mathrm{mU} / \mathrm{l}$ oder sogar $1,0 \mathrm{mU} / \mathrm{l}$ genannt. Woher stammen aber diese Grenzwerte?

In der Tat gibt es Studien, die eine gesteigerte Prävalenz latenter Hypothyreosen und erhöhter Schilddrüsenautoantikörperkonzentrationen bei Sterilitätspatientinnen sowie eine geringere Schwangerschaftsrate bei Sterilitätstherapien aufzeigen. Auch konnte belegt werden, dass die Behandlung einer latenten Hypothyreose zu normalen Schwangerschaftsund Geburtenraten führt [11]. Aufgrund dieser und anderer Studien ist eine Schilddrüsensubstitution zweifelsohne sinnvoll, wenn eine latente Hypothyreose vorliegt, d. h. TSH-Werte über etwa 4,0 mU/l und normale Konzentrationen des freien Trijodthyronins $\left(\mathrm{T}_{3}\right)$ und Thyroxins $\left(\mathrm{T}_{4}\right)$.

\section{》) Eine Schilddrüsenhormon- substitution sollte nicht nach dem Gießkannenprinzip erfolgen}

Aber gilt dies auch für TSH-Werte knapp unter dem oberen Grenzwert? Bei einer In-vitro-Fertilisation (IVF) steigt der
TSH-Wert etwas an. Besonders ausgeprägt ist die Zunahme bei einer Überstimulation und bei erhöhten Schilddrüsenautoantikörperkonzentrationen. Dieser Effekt hält mehrere Monate an [7]. Berücksichtigt man, dass der Bedarf an Schilddrüsenhormonen in der Schwangerschaft zusätzlich auch natürlicherweise ansteigt, ist eine klinisch relevante Schilddrüsendysfunktion nach einer IVF denkbar. Daher sollte der TSH-Wert vor einer IVF-Therapie auf $<2,5 \mathrm{mU} / \mathrm{l}$ gesenkt werden [1], insbesondere bei erhöhten Schilddrüsenautoantikörpern. In Bezug auf Konstellationen, in denen keine IVF-Therapie durchgeführt wird, ist die Datenlage jedoch weitgehend unklar. Entsprechend ist die Relevanz des Grenzwerts in diesen Fällen fraglich. Liegt der TSH-Wert bei $>2,5 \mathrm{mU} / \mathrm{l}$, aber nicht im Normbereich, so ist es eine Möglichkeit, zunächst den oft schwankenden TSH-Wert zu kontrollieren und nur bei persistierend erhöhten TSH-Werten zu substituieren. Wird eine Substitution eingeleitet, so sollte sie bis zur Geburt fortgeführt und dann wieder abgesetzt werden.

\section{Fazit}

Ein latente Hypothyreose (TSH über etwa 4,0 mU/l) sollte immer behandelt werden. Bei einem persistierenden TSH-Wert von $2,5-4,5 \mathrm{mU} / \mathrm{l}$ ist bei einer IVF-Therapie mit Gonadotropinstimulation - insbesondere bei erhöhten Schilddrüsenautoantikörperkonzentrationen - eine $\mathrm{T}_{4}$-Gabe, z. B. $50 \mu \mathrm{g} / \mathrm{Tag}$, sinnvoll. Wird keine IVF durchgeführt, ist der Nutzen einer Substitution sehr fraglich.

\section{Schilddrüsenfunktion in \\ Peri- und Postmenopause}

Im Rahmen des Alterungsprozesses der Hypothalamus-Hypophysen-ThyroideaAchse werden verschiedene Veränderungen beschrieben. So steigt etwa, zumindest in Gebieten ohne Jodmangel, die TSH-Konzentration im Serum leicht an. In der Folge wird häufiger die Diagnose einer subklinischen Hypothyreose gestellt. Möglicherweise gelten aber im höheren Alter andere Referenzwerte, sodass viele Patienten mit Diagnose einer subklinischen Hypothyreose übertherapiert wä- ren [15]. Andererseits ist ein TSH-Wert im Serum >10 mU/l häufiger mit kardiovaskulären Risiken verbunden [12]. Somit gilt als Therapieindikation bei subklinischer Hypothyreose weiterhin ein TSH-Spiegel $>10 \mathrm{mU} / \mathrm{l}$; darunter bleibt die Entscheidung individuell.

Bei Frauen mit therapierter (subklinischer) Hypothyreose steigt bei Anwendung oraler Östrogene der $\mathrm{T}_{4}$-Bedarf. Daher sollte ab dem Beginn der Östrogentherapie alle 6 Wochen eine Kontrolle von $\mathrm{TSH}, \mathrm{fT}_{4}$ und des $\mathrm{T}_{4}$-bindenden Globulins (TBG) im Serum erfolgen und ggf. die $\mathrm{T}_{4}$-Dosis angepasst werden [8].

\section{Fazit}

Die Höhe der Therapieschwelle bei einer subklinischen Hypothyreose ist umstritten. Frauen mit therapierter (subklinischer) Hypothyreose sollten bei Beginn einer oralen Östrogentherapie beobachtet werden, die $\mathrm{T}_{4}$-Dosis ist ggf. anzupassen.

\section{Vitamin-D-Supplementation}

Calcitriol (1a,25-Dihydroxy-Vitamin $\mathrm{D}_{3}$ ) ist die aktive Form von Vitamin D und u. a. für die Mineralisierung des Knochens zuständig. Zusammen mit Kalzium reduziert Vitamin D das Frakturrisiko z. B. bei Heimbewohnern [3]. Als Maß für die gesamte Vitamin-D-Exposition des Körpers gilt aber Calcifediol (25-Hydroxy-Vitamin $\mathrm{D}_{3}$ ).

Bei der Beurteilung des Frakturrisikos erfolgt oft keine Differenzierung zwischen der Vitamin-D-Dosierung und dem tatsächlich erreichten 25-Hydroxy-Vitamin$\mathrm{D}_{3}$-Spiegel im Serum. Zudem gibt es kein einheitliches Verfahren zur Bestimmung von Vitamin D. Eine einheitliche Definition des Vitamin-D-Mangels wird dadurch erschwert. Eine Möglichkeit ist die Unterteilung in eine mangelhafte $(<10 \mathrm{ng} /$ $\mathrm{ml} \mathrm{bzw.}<25 \mathrm{nmol} / \mathrm{l})$, ungenügende (10$30 \mathrm{ng} / \mathrm{ml}$ bzw. 25-75 nmol/l) und adäquate Vitamin D-Versorgung (>30 ng/ $\mathrm{ml}$ bzw. $>75 \mathrm{nmol} / \mathrm{l})$.

Die diätetischen Empfehlungen internationaler Fachgesellschaften zur Vitamin-D- bzw. Cholecalciferolsupplementation bei Frauen unterscheiden sich. Sie reichen von $600 \mathrm{IE} / \mathrm{Tag}$ (Alter $<70$ Jahre) 
bis $800 \mathrm{IE} / \mathrm{Tag}$ ( $\geq 70$ Jahre; Institute of Medicine) oder liegen altersunabhängig bei 1500-2000 IE/Tag [4]. Die Vitamin-DSupplementation bei postmenopausaler Osteoporose kann täglich (800 IE/Tag), wöchentlich (5600 IE/Woche) oder monatlich (24.000 IE/Monat) durchgeführt werden, sollte aber aufgrund eines ansonsten erhöhten Sturz- und Frakturrisikos nicht jährlich erfolgen. Dosierungen bis $2000 \mathrm{IE} / \mathrm{Tag}$ erscheinen sicher. Eine (Verlaufs-)Kontrolle von 25-Hydroxy-Vitamin $\mathrm{D}_{3}$ im Serum (nach 3 Monaten) ist bei erhöhtem Risiko zu empfehlen, so etwa bei Osteoporose, Malabsorption, Adipositas, älteren Patienten mit Sturzanamnese oder bei Medikamentenanwendung, z. B. von Antikonvulsiva, Steroiden oder hochaktiven antiretroviralen Therapeutika.

\section{Fazit}

\section{Auf eine adäquate Versorgung mit Vita- min D ist v. a. in Risikopopulationen zu achten.}

\section{North American Menopause Society (NAMS)}

\section{Das koronare „window of opportunity"}

Die sog. Timing-Hypothese besagt, dass bei einem Beginn der Hormonersatztherapie (HRT) vor dem 60. Lebensjahr bzw. innerhalb von 10 Jahren nach der Menopause ein günstiger Effekt auf das Herz zu erwarten ist.

In der randomisierten, placebokontrollierten Kronos Early Estrogen Prevention Study (KEEPS) wurden 727 symptomatische Frauen in der frühen Postmenopause und im Alter von 42-59 Jahren über 4 Jahre mit transdermalem Östradiol $(50 \mu \mathrm{g} / \mathrm{Tag})$, oralen konjugierten equinen Östrogenen $(0,45 \mathrm{mg} / \mathrm{Tag})$ oder Placebo behandelt. Die aktiven Behandlungsarme erhielten außerdem zyklisch mikronisiertes Progesteron (200 mg/Tag an 12 Tagen pro Monat).

Es zeigte sich ein grundsätzlich vorteilhafter Effekt der Östrogene auf bekannte Risikofaktoren für eine koronare Herzkrankheit. Bezüglich der Karotis-IntimaMedia-Dicke und des Koronarkalks ergab sich jedoch bei Therapieende kein Gruppenunterschied. In keinem Behandlungsarm kam es zu einem signifikanten Anstieg schwerer unerwünschter Ereignisse (venöse Thromboembolie, Apoplex, Herzinfarkt, Mammakarzinom, Mortalität).

\section{Fazit}

KEEPS konnte die Timing-Hypothese bisher nicht belegen. Eine 4-jährige HRT in der frühen Postmenopause ist nicht mit signifikanten Risiken verbunden.

\section{Erfassung kardiovaskulärer Risiken bei asymptomatischen Frauen}

Seit 1984 sterben in den USA mehr Frauen als Männer an kardiovaskulären Erkrankungen. Als Screeningtool für asymptomatische Frauen wird dort der Framingham Risk Score empfohlen, der das 10-Jahres-Risiko für kardiovaskuläre Erkrankungen aus den Parametern Alter, Cholesterin, Blutdruck, Diabetes mellitus und Nikotinabusus errechnet. Ein entsprechendes Instrument in der Schweiz ist der AGLA-Risikoscore (http://www.agla. ch/risikoberechnung/agla-risikorechner). Bei moderatem oder hohem errechnetem Risiko wird die Vorstellung beim Hausarzt oder Kardiologen empfohlen.

Die allgemeinen Empfehlungen der American Heart Association für Herzgesundheit umfassen Lebensstilinterventionen (Bewegung, Ernährung, Nikotinverzicht), Gewichtskontrolle, ggf. $\omega$-3-Fettsäuren (Eicosapentaensäure $1800 \mathrm{mg} /$ Tag) sowie bei Bedarf die medikamentöse Blutdruck-, Lipid- und Glukosekontrolle. Eine HRT, Antioxidanzien (Vitamin E und $\mathrm{C}, \beta$-Karotin), Folsäure (Ausnahme: reproduktives Alter), Vitamin $\mathrm{B}_{6}, \mathrm{~B}_{12}$ und Acetylsalicylsäure bei gesunden Frauen $<65$ Jahren werden nicht empfohlen [9].

\section{Neue medikamentöse Therapieansätze bei Adipositas}

Es gibt eine Behandlungslücke zwischen einer Diät bzw. Lifestyle-Modifikation, die sich bei vielen als wenig effektiv erweist, und der bariatrischen Chirurgie, die für viele zu riskant ist.
$\mathrm{Zu}$ den neuen Pharmakotherapeutika bei Adipositas zählen seit 2012 in den USA Lorcaserin (Belviq ${ }^{\circledast}$ ) und Phentermin plus Topiramat (Qsymia $\left.{ }^{\circledast}\right)$. Lorcaserin ist ein selektiver $5-\mathrm{HT}_{2 \mathrm{c}}$-Rezeptor-Agonist. Die Indikationen sind zum einen ein BodyMass-Index $(\mathrm{BMI})>30 \mathrm{~kg} / \mathrm{m}^{2}$ und zum anderen ein $\mathrm{BMI}>27 \mathrm{~kg} / \mathrm{m}^{2}$ plus mindestens eine gewichtsbedingte Komorbidität (Hypertonie, Diabetes mellitus Typ 2, Dyslipidämie).

In der Zulassungsstudie zu Lorcaserin zeigte sich ein Gewichtsverlust von $>5 \%$ des Ausgangsgewichts bei 47,5\% (Lorcaserin) vs. 20,3\% der Patienten (Placebo) nach einem Jahr. Der absolute Gewichtsverlust betrug nach einem Jahr 5,8 kg (Lorcaserin) vs. 2,2 kg (Placebo). Erfolgreiche Lorcaserin-Anwender im ersten Jahr konnten ihr Gewicht im zweiten Jahr mit Lorcaserin (68\%) besser halten als mit Placebo (50\%). Zu den Nebenwirkungen zählen Übelkeit, Kopfschmerzen und Schwindel [14].

Die Kombination aus Phentermin, das die Freisetzung von Katecholaminen fördert, und dem $\gamma$-Aminobuttersäure(GABA)-ergen Topiramat wurde für dieselbe Indikation wie Lorcaserin zugelassen. Es ist in der Schwangerschaft kontraindiziert. In der Zulassungsstudie zeigte sich ein Gewichtsverlust von $>5 \%$ des Ausgangsgewichts bei 67\% (Phentermin/ Topiramat hoch dosiert) vs. $45 \%$ (Phentermin/Topiramat niedrig dosiert) vs. $17 \%$ (Placebo). Zu den Nebenwirkungen zählen Mundtrockenheit, Parästhesie, Obstipation und Schlafstörungen [2].

Zur Unterstützung der Therapieindikation und -wahl wurde vor einigen Jahren eine multifaktorielle Klassifikation der Adipositas vorgeschlagen, die nicht nur den BMI und Bauchumfang berücksichtigt, das sog. Edmonton Obesity Staging System [13].

\section{Fazit}

In den USA sind seit 2012 neue Pharmakotherapien mit strenger Indikationsstellung für die Behandlung der Adipositas zugelassen. Dadurch soll die Therapielücke zwischen Diät und bariatrischer Chirurgie geschlossen werden. 


\section{Kein Überlebensvorteil durch eine prophylaktische bilaterale Ovarektomie}

In der Nurses Health Study werden $>100.000$ Krankenschwestern seit 1976 alle 2 Jahre per postalischem Fragebogen untersucht. Hiervon wurden 16.914 aufgrund eines benignen Befunds einer Hysterektomie und prophylaktischen beidseitigen Ovarektomie unterzogen und mit 13.203 Frauen mit alleiniger Hysterektomie verglichen. Der Beobachtungszeitraum beträgt 28 Jahre.

Es zeigte sich ein geringeres Risiko, an einem Ovarialkarzinom zu sterben [Hazard Ratio (HR): 0,06; 95\%-Konfidenzintervall (KI): 0,02-0,17]. Ebenso war das Risiko geringer, an einem Mammakarzinom zu sterben, wenn die Ovarektomie in einem Alter $<47,5$ Jahren durchgeführt wurde $(\mathrm{p}=0,048)$. Unabhängig vom Alter bei der Operation war das Risiko erhöht, an folgenden Ursachen zu sterben: Herz-Gefäß-Erkrankung (HR: 1,23; 95\%KI: 1,00-1,52), Bronchialkarzinom (HR: 1,29; 95\%-KI: 1,04-1,61), kolorektales Karzinom (HR: 1,49; 95\%-KI: 1,02-2,18), alle Krebserkrankungen (HR: 1,16; 95\%KI: 1,05-1,29) und alle Ursachen zusammengenommen (HR: 1,13; 95\%-KI: 1,061,21). Für Frauen, die vor dem 50. Lebensjahr prophylaktisch beidseitig ovarektomiert wurden und dann eine Östrogentherapie erhielten, war die Gesamtmortalität jedoch nicht erhöht (HR: 1,05; 95\%KI: 0,94-1,17; [10]).

\section{Fazit}

\section{In keinem Alter ist die prophylaktische beidseitige Ovarektomie mit einem Überlebensvorteil verbunden.}

\section{Ausblick}

Aufgrund der großen Resonanz mit 150 Teilnehmern und sehr guten Evaluationsergebnisse wird die Veranstaltung auch im Jahr 2014 wieder in Olten im Stadttheater stattfinden. Geplanter Termin der Veranstaltung ist der 11.09.2014 von etwa 14:00-18:00 Uhr. Details finden sich auf der Website (http://www.weltkongresse.ch).

\section{Korrespondenzadresse}

Prof. Dr. M. von Wolff

Abteilung für Gynäkologische Endokrinologie und Reproduktionsmedizin, Universitäts-Frauenklinik, Inselspital Bern

Effingerstr. 102, 3010 Bern

Schweiz

michael.vonwolff@insel.ch

\section{Einhaltung ethischer Richtlinien}

Interessenkonflikt. M. von Wolff und P. Stute geben an, dass kein Interessenkonflikt besteht.

Dieser Beitrag beinhaltet keine Studien an Menschen oder Tieren.

\section{Literatur}

1. Abalovich M, Amino N, Barbour LA et al (2007) Management of thyroid dysfunction during pregnancy and postpartum: an Endocrine Society Clinical Practice Guideline. J Clin Endocrinol Metab 92(8 Suppl):S1-S47

2. Allison DB, Gadde KM, Garvey WT et al (2012) Controlled-release phentermine/topiramate in severely obese adults: a randomized controlled trial (EQUIP). Obesity (Silver Spring) 20:330-342

3. Chung M, Lee J, Terasawa T (2011) Vitamin D with or without calcium supplementation for prevention of cancer and fractures: an updated meta-analysis for the U.S. Preventive Services Task Force. Ann Intern Med 155:827-838

4. Holick MF, Binkley NC, Bischoff-Ferrari HA et al (2012) Guidelines for preventing and treating vitamin D deficiency and insufficiency revisited. J Clin Endocrinol Metab 97:1153-1158

5. Kaur G, Phillips C, Wong K, Saini B (2013) Timing is important in medication administration: a timely review of chronotherapy research. Int J Clin Pharm 35:344-358

6. Kerdelhué B, Brown S, Lenoir V et al (2002) Timing of initiation of the preovulatory luteinizing hormone surge and its relationship with the circadian cortisol rhythm in the human. Neuroendocrinology $75: 158-163$

7. Krassas GE, Poppe K, Glinoer D (2010) Thyroid function and human reproductive health. Endocr Rev 31:702-755

8. Mazer NA (2004) Interaction of estrogen therapy and thyroid hormone replacement in postmenopausal women. Thyroid 14(Suppl 1):S27-S34

9. Mosca L, Benjamin EJ, Berra K et al (2011) Effectiveness-based guidelines for the prevention of cardiovascular disease in women - 2011 update: a guideline from the American Heart Association. Circulation 123:1243-1262

10. Parker WH, Feskanich D, Broder MS et al (2013) Long-term mortality associated with oophorectomy compared with ovarian conservation in the nurses' health study. Obstet Gynecol 121:709-716

11. Raber W, Nowotny P, Vytiska-Binstorfer E, Vierhapper $\mathrm{H}$ (2003) Thyroxine treatment modified in infertile women according to thyroxine-releasing hormone testing: 5 year follow-up of 283 women referred after exclusion of absolute causes of infertility. Hum Reprod 18:707-714
12. Rodondi N, Elzen WP den, Bauer DC et al (2010) Subclinical hypothyroidism and the risk of coronary heart disease and mortality. JAMA 304:13651374

13. Sharma AM, Kushner RF (2009) A proposed clinical staging system for obesity. Int J Obes (Lond) 33:289-295

14. Smith SR, Weissman NJ, Anderson CM et al (2010) Multicenter, placebo-controlled trial of lorcaserin for weight management. N Engl J Med 363:245256

15. Surks MI, Hollowell JG (2007) Age-specific distribution of serum thyrotropin and antithyroid antibodies in the US population: implications for the prevalence of subclinical hypothyroidism. J Clin Endocrinol Metab 92:4575-4582

16. WHO (1980) Temporal relationships between ovulation and defined changes in the concentration of plasma estradiol-17 beta, luteinizing hormone, follicle-stimulating hormone, and progesterone. I. Probit analysis. World Health Organization, Task Force on Methods for the Determination of the Fertile Period, Special Programme of Research, Development and Research Training in Human Reproduction. Am J Obstet Gynecol 138:383-390 